\title{
Antiplane Deformations for
}

Anisotropic Multilayered Media by Using the Coordinate Transform Method

Ru-Li Lin

Graduate Student

Chien-Ching Ma

Professor

Department of Mechanical Engineering, National Taiwan University, Taipei, Taiwan 10617 R.O.C.
Green's functions for anisotropic elastic multilayered media subjected to antiplane shear deformation are presented in this study. The antiplane shear deformation due to a concentrated shear force and screw dislocation in an arbitrary layer was investigated in detail. A linear coordinate transformation is introduced in this study to simplify the problem. The linear coordinate transformation reduces the anisotropic multilayered problem to an equivalent isotropic problem without complicating the geometry of the problem. Explicit analytical solutions were derived using the Fourier transform and the series expansion technique. The complete solutions for the multilayered problem consist only of the simplest solutions obtained from an infinite homogeneous medium with concentrated loadings. Numerical results for the full-field stress distribution in multilayered media subjected to a point body force are presented. These numerical results were compared with the solutions obtained by considering the multilayered medium as one layer with effective elastic constants determined from the averaged material constants of the multilayered medium. It is found that the shear stress $\tau_{y z}$ of the homogeneous one layer solution is a very good approximation of the result for the multilayered medium; however, the shear stress $\tau_{x z}$ in these two solutions has a large discrepancy due to the fact that $\tau_{x z}$ is discontinuous at the interfaces of the multilayered medium. [S0021-8936(00)01703-7]

\section{Introduction}

Antiplane shear deformations are the simplest of twodimensional deformations that arise in anisotropic or isotropic elastic bodies. For the antiplane shear deformation, the displacement is parallel to the axial coordinate that is normal to the plane and is dependent only on the coordinates in the plane. Such deformation field characterized by a single axial displacement can be regarded as complementary to that of plane-strain deformation (e.g., [1]). The antiplane problem plays a useful role as a pilot problem that reveals simpler aspects of elasticity solutions. The antiplane problem of two dissimilar anisotropic wedges has been considered by Ma [2] and Ma and Hour [3] using the Mellin transform method. They showed that the stress and displacement fields have reduced dependence on the elastic constants. Retting [4] did the antiplane stress analysis of a cracked beam made of orthotropic material. Wu and Chiu [5] discussed interface cracks in anisotropic bimaterials subjected to antiplane shearing. Ting [6] also provided many basic discussions and investigated some fundamental problems for anisotropic antiplane deformations. In addition, considerable attention has been paid to the analysis of antiplane shear deformation in nonlinear elasticity theory for isotropic solids, cf. Jiang and Knowles [7], Polignone and Horgan [8] and the references therein. A comprehensive review of antiplane shear for both linear and nonlinear elasticity is given by Horgan [9].

Analysis of anisotropic elasticity problems is often tedious due to the presence of many elastic constants. It is desirable to reduce the dependence on elastic constants through theoretical consider-

Contributed by the Applied Mechanics Division of THE AMERICAN SOCIETY OF MECHANICAL ENGINEERS for publication in the ASME JOURNAL OF APPLIED MECHANICS. Manuscript received by the ASME Applied Mechanics Division, May 3, 1999; final revision, Nov. 30, 1999. Associate Technical Editor: M.-J. Pindera. Discussion on the paper should be addressed to the Technical Editor, Professor Lewis T. Wheeler, Department of Mechanical Engineering, University of Houston, Houston, TX 77204-4792, and will be accepted until four months after final publication of the paper itself in the ASME JOURNAL OF APPLIED MECHANICS. ations in advance of the analysis of a given boundary value problem. For anisotropic elasticity, Lekhnitskii's formulation ([10]) and Stroh's formulation ([11]) are the two widely used methods. The general solutions obtained by these methods showed that the antiplane anisotropic problem can be converted to a corresponding isotropic problem by properly changing the geometry of the original configuration and the tractions on the boundary. In other words, the anisotropic antiplane problem can be simplified to an isotropic problem with the aid of a suitable coordinate transformation. In the isotropic case, the displacement equilibrium equation becomes the Laplace equation which can be solved easily. For the anisotropic problem, however, the governing equilibrium equation is a general second-order partial differential equation with constant coefficients. In particular, a variety of coordinate transformations could be used to convert the general second-order partial differential equation to the Laplace equation. The properties of the coordinate transformations for antiplane deformations have been investigated by Ma [12] and Horgan and Miller [1]. An orthotropic transformation concept was introduced by Yang and Ma [13] to analyze the much complicated and difficult in-plane deformations for planar anisotropic solids.

Because of the rapid expansion in the use of structural components made of laminated materials, predictions of the behavior of multilayered media subjected to arbitrary loads are needed. The stress states at the interfaces of multilayered media are of particular interest because delamination may occur if allowable levels are exceeded. For multilayered anisotropic media, the problem becomes more complicated than that of homogeneous isotropic counterparts. Based on the mixed formulation of elasticity, Bufler [14] and Bahar [15] independently constructed the transfer matrix approach to solve multilayered media problems. A local/global stiffness matrix approach which is similar to the transfer matrix approach was employed by Pindera [16] and Pindera and Lane $[17,18]$ to solve round punch contact problems of arbitrarily lami- 
nated media. The local/global stiffness matrix method is based on the systematic construction of a global stiffness matrix in terms of local stiffness matrices of the individual layers.

According to the classical Saint-Venant principle, the precise decay factor of the stress with distance from the region of applied load depends on the geometry of the body, the boundary conditions, the applied load and material properties. For layered media, this issue has been examined by Baxter and Horgan $[19,20]$. They showed that material inhomogeneity significantly affects the practical application of Saint-Venant's principle to sandwich structures. Wu and Chiu [21] have solved the problem of a semiinfinite multilayered monoclinic strip loaded by shear stress at the end by using eigenfunction expansion in conjunction with Betti's reciprocity theorem.

In the current study, an anisotropic elastic multilayered medium with $n$ layers subjected to antiplane loading within an arbitrary layer is investigated. The material properties and the thickness in each layer are different. One of the objectives of this study is to develop an effective analytical methodology to construct the fullfield solutions for this complicated problem. A general linear coordinate transformation is introduced in this study to simplify the problem. This linear coordinate transformation will simplify the governing equilibrium equation without complicating the boundary and interface continuity conditions. Based on this transformation, the original anisotropic multilayered problem is converted to an equivalent isotropic multilayered problem. The analytical solutions for the stresses and displacement obtained in this study are exact and are expressed in an explicit closed form. For a numerical example, a multilayered medium with 12 layers is discussed in detail. The stress distribution for the multilayered solution is compared with the homogeneous single layer solution by averaging the material constants of the multilayered medium.

\section{Antiplane Shear Deformations of an Anisotropic Elastic Solid}

In the absence of body forces, the equilibrium equations for the elastostatic problem are

$$
\sigma_{i j, j}=0
$$

where the repeated indices imply summation and a comma stands for differentiation. The generalized Hooke's law for an anisotropic, homogeneous, and linearly elastic solid is given by

$$
\sigma_{i j}=C_{i j k l} \varepsilon_{k l},
$$

where $\varepsilon_{k l}=1 / 2\left(u_{k, l}+u_{l, k}\right)$ denotes the infinitesimal strain tensor, and $C_{i j k l}$ are the elastic stiffnesses satisfying the usual symmetry conditions. Due to the symmetry of $C_{i j k l}$, Eq. (2) can be rewritten as

$$
\sigma_{i j}=C_{i j k l} u_{k, l} .
$$

For a general anisotropic elastic material, an antiplane deformation or a plane deformation in general does not exist. For some special anisotropic materials possessing elastic stiffnesses $C_{i j k l}$ which are written in a contracted notation in the form

$$
\mathbf{C}=\left[\begin{array}{cccccc}
C_{11} & C_{12} & C_{13} & 0 & 0 & C_{16} \\
& C_{22} & C_{23} & 0 & 0 & C_{26} \\
& & C_{33} & C_{34} & C_{35} & C_{36} \\
& & & C_{44} & C_{45} & 0 \\
& \text { sym. } & & & C_{55} & 0 \\
& & & & & C_{66}
\end{array}\right],
$$

the plane and antiplane deformations will be uncoupled and such anisotropic elastic materials are capable of an antiplane deformation (see, e.g., Horgan and Miller [1], Ting [6]).

For two-dimensional problems, the Cartesian coordinate system is chosen such that the antiplane deformation is in the $z$-direction.
Let $u, v$, and $w$, respectively, represent the displacement components in the $x, y$, and $z$-direction of the Cartesian coordinate system. For antiplane shear deformations,

$$
u=v=0, \quad w=w(x, y),
$$

and the relevant shear stresses are denoted by $\tau_{y z}$ and $\tau_{x z}$. If the material constants have the form represented in Eq. (4), then the equilibrium equations in the $x$ and $y$-directions are automatically satisfied, and the equilibrium equation in the $z$-direction can be written in terms of the displacement $w$ as

$$
C_{55} \frac{\partial^{2} w}{\partial x^{2}}+2 C_{45} \frac{\partial^{2} w}{\partial x \partial y}+C_{44} \frac{\partial^{2} w}{\partial y^{2}}=0 .
$$

Equation (6) is the governing equation for an anisotropic antiplane deformation problem, and is a homogeneous second-order partial differential equation for displacement $w$. The nonzero stresses are related to the displacement as follows:

$$
\begin{gathered}
\tau_{y z}=C_{45} \frac{\partial w}{\partial x}+C_{44} \frac{\partial w}{\partial y}, \\
\tau_{x z}=C_{55} \frac{\partial w}{\partial x}+C_{45} \frac{\partial w}{\partial y}, \\
\sigma_{z z}=C_{35} \frac{\partial w}{\partial x}+C_{34} \frac{\partial w}{\partial y} .
\end{gathered}
$$

For monoclinic materials with the plane of symmetry $z=0, C_{34}$ $=C_{35}=0$ so that $\sigma_{z z}=0$.

\section{Linear Coordinate Transformation}

The governing equation expressed in Eq. (6) is a general homogeneous second-order partial differential equation with constant coefficients. Such a linear partial differential equation can be transformed into the Laplace equation by a linear coordinate transformation (see, e.g., Horgan and Miller [1]). A special linear coordinate transformation is introduced as

$$
\left[\begin{array}{l}
X \\
Y
\end{array}\right]=\left[\begin{array}{ll}
1 & \alpha \\
0 & \beta
\end{array}\right]\left[\begin{array}{l}
x \\
y
\end{array}\right],
$$

where $\alpha=-C_{45} / C_{44}, \beta=\mu^{e} / C_{44}$ and $\mu^{e}=\sqrt{C_{44} C_{55}-C_{45}^{2}}$. Assume that $C_{44}$ and $C_{55}$ as well as $\sqrt{C_{44} C_{55}-C_{45}^{2}}$ are all positive. After the coordinate transformation, Eq. (6) can be rewritten as the standard Laplace equation in the $(X, Y)$ coordinate system

$$
\mu^{e}\left(\frac{\partial^{2} w}{\partial X^{2}}+\frac{\partial^{2} w}{\partial Y^{2}}\right)=0
$$

It is interesting to note that the mixed derivative disappears from Eq. (6). The relationships between the shear stresses in the two coordinate systems are given by

$$
\begin{gathered}
\tau_{y z}(x, y)=\mu^{e} \frac{\partial w(X, Y)}{\partial Y}=\tau_{Y Z}(X, Y), \\
\tau_{x z}(x, y)=\beta \mu^{e} \frac{\partial w(X, Y)}{\partial X}-\alpha \mu^{e} \frac{\partial w(X, Y)}{\partial Y} \\
=\beta \tau_{X Z}(X, Y)-\alpha \tau_{Y Z}(X, Y) .
\end{gathered}
$$

In a mathematical sense, Eqs. (6) and (7) are transformed to Eqs. (9) and (10) by the linear coordinate transformation expressed in Eq. (8), or in a physical sense, the governing Eq. (6) and the stress displacement relation (7) of an anisotropic antiplane problem are converted into an equivalent isotropic problem by properly changing the geometry of the body using the linear coordinate transformation, Eq. (8). The coordinate transformation in Eq. (8) has the following characteristics: (a) it is linear and continuous, (b) an anisotropic problem is converted to an isotropic problem after the transformation, and (c) there is no stretching and rotation in the 


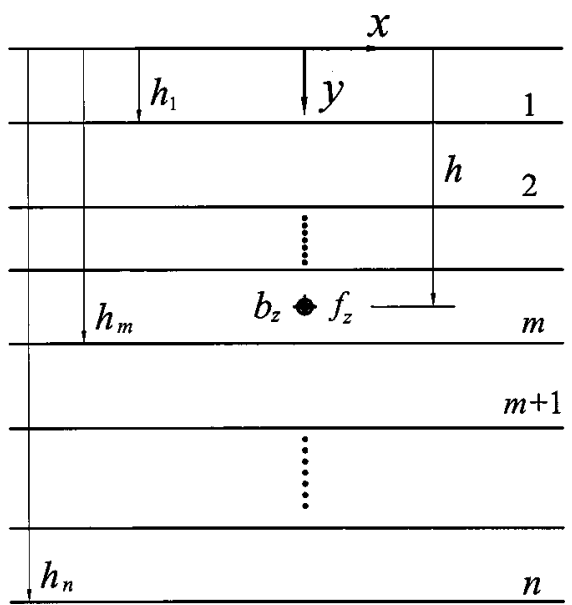

Fig. 1 Configuration and coordinates system of an anisotropic multilayered medium

horizontal direction. These important features offer advantages in dealing with straight boundaries and interfaces in the multilayered system discussed in the present study. The most interesting feature is that a straight line $\left(x_{1}, y_{0}\right),\left(x_{2}, y_{0}\right)$ that is parallel to the $x$-axis will remain a straight line $\left(X_{1}, Y_{0}\right),\left(X_{2}, Y_{0}\right)$ parallel to the $X$-axis after the transformation, and the length of the line will not change, i.e., $X_{2}-X_{1}=x_{2}-x_{1}$. The relationship between the anisotropic problem and the corresponding isotropic problem in the polar coordinate system and more detailed discussions of this linear coordinate transformation were presented by $\mathrm{Ma}$ [12].

The linear coordinate transformation described by Eq. (8) can be used to solve the anisotropic antiplane problem for only a single material. However, for a multilayered anisotropic medium with straight interfaces shown in Fig. 1, a modification of the linear coordinate transformation will be introduced in the following section to transform the multilayered anisotropic problem to an equivalent multilayered isotropic problem.

\section{Formulations for Multilayered Media Subjected to Interior Loadings}

In the following sections, the Green's function for an anisotropic $n$-layered medium will be constructed. Consider an anisotropic $n$-layered medium with $n$ layers subjected to a line of constant force $f_{z}$ and a screw dislocation of magnitude $b_{z}$ along the $z$-axis located in the $m$ th layer. The displacement and shear stresses are independent of the $z$-axis and so we can consider this problem as a two-dimensional antiplane problem. In other words, the original problem can be simplified as a two-dimensional multilayered medium subjected to a point shear force and a screw dislocation shown in Fig. 1. The displacement equilibrium equation in each layer is expressed as

$$
C_{55}^{j} \frac{\partial^{2} w^{j}}{\partial x^{2}}+2 C_{45}^{j} \frac{\partial^{2} w^{j}}{\partial x \partial y}+C_{44}^{j} \frac{\partial^{2} w^{j}}{\partial y^{2}}=0, \quad j=1,2, \ldots, n .
$$

The boundary conditions on the top and bottom surfaces of the layered medium are

$$
\tau_{y z}^{1}(x, 0)=0, \quad \tau_{y z}^{n}\left(x, h_{n}\right)=0 .
$$

The jump conditions for the shear stress and displacement across the point loads within the $m$ th layer are

$$
\begin{gathered}
\tau_{y z}^{m^{+}}\left(x, h^{+}\right)-\tau_{y z}^{m^{-}}\left(x, h^{-}\right)=-f_{z} \delta(x), \\
w^{m^{+}}\left(x, h^{+}\right)-w^{m^{-}}\left(x, h^{-}\right)=-b_{z} U(x),
\end{gathered}
$$

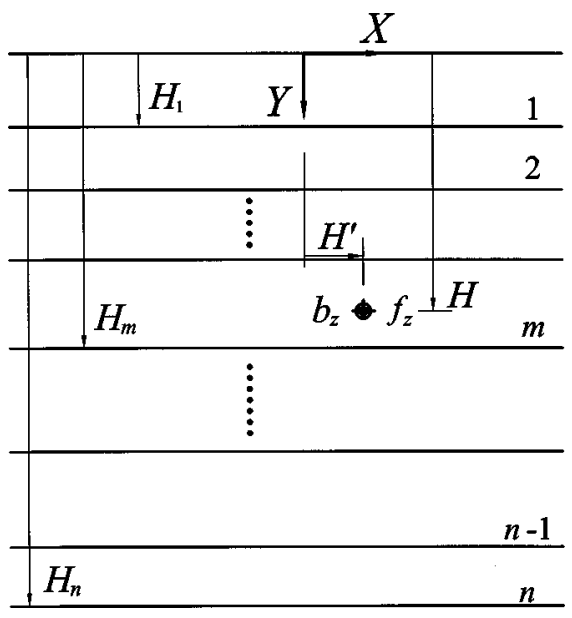

Fig. 2 Configuration and coordinates system for the multilayered medium after the linear coordinate transformation

where $\delta()$ is the delta function and $U()$ is the heaviside function. In Eq. (13), $\tau_{y z}^{m^{+}}$and $\tau_{y z}^{m^{-}}$indicate the shear stress above and below the plane of applied loadings in the $m$ th layer; $h^{+}$and $h^{-}$ denote the position just above and below the applied loadings at $y=h$ (see Fig. 1). Application of the traction and displacement continuity conditions at the interface between the $j$ th and $j+1$ th layer, yields

$$
\begin{aligned}
& \tau_{y z}^{j}\left(x, h_{j}\right)=\tau_{y z}^{j+1}\left(x, h_{j}\right), \\
& w^{j}\left(x, h_{j}\right)=w^{j+1}\left(x, h_{j}\right), \quad j=1,2, \ldots, n-1 .
\end{aligned}
$$

In order to maintain the geometry of the layered configuration, the linear coordinate transformation described in Eq. (8) is modified for each layer as follows:

$$
\left[\begin{array}{c}
X \\
Y
\end{array}\right]=\left[\begin{array}{cc}
1 & \alpha_{j} \\
0 & \beta_{j}
\end{array}\right]\left[\begin{array}{l}
x \\
y
\end{array}\right]+\sum_{k=1}^{j-1} h_{k}\left[\begin{array}{c}
\alpha_{k}-\alpha_{k+1} \\
\beta_{k}-\beta_{k+1}
\end{array}\right], \quad j=1,2, \ldots, n .
$$

Comparing with Eq. (8), the first term on the right-hand side of Eq. (15) retains exactly the same form while the second summation term becomes the modified term. The new coordinate transformation possesses the following characteristics: (a) no gaps or overlaps are generated along the interface and (b) no sliding and no mismatches occur along the interface. The geometric configuration in the transformed $(X, Y)$ coordinate is shown in Fig. 2. Note that while the thickness of each layer is changed, the interfaces are parallel to the $X$-axis. Thus, the new geometric configuration is similar to the original problem.

The equilibrium equations in the transformed coordinate are governed by the standard Laplace equation expressed by

$$
\mu_{j}^{e} \frac{\partial^{2} w^{j}}{\partial X^{2}}+\mu_{j}^{e} \frac{\partial^{2} w^{j}}{\partial Y^{2}}=0
$$

The displacement $w$ and the shear stress $\tau_{Y Z}$ are continuous along the interfaces in the transformed coordinates,

$$
\begin{gathered}
w^{j}\left(X, H_{j}\right)=w^{j+1}\left(X, H_{j}\right), \quad \tau_{Y Z}^{j}\left(X, H_{j}\right)=\tau_{Y Z}^{j+1}\left(X, H_{j}\right), \\
j=1,2, \ldots, n-1,
\end{gathered}
$$

where

$$
H_{j}=\beta_{j} h_{j}+\sum_{k=1}^{j-1}\left(\beta_{k}-\beta_{k+1}\right) h_{k} .
$$

The top and bottom surfaces are traction free and can be expressed as

$$
\tau_{Y Z}^{1}(X, 0)=0, \quad \tau_{Y Z}^{n}\left(X, H_{n}\right)=0 .
$$




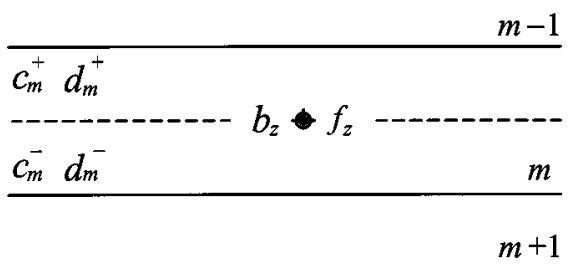

Fig. 3 The jump conditions for applied loadings at the $m$ th layer

The jumps within the $m$ th layer caused by the applied antiplane body force and screw dislocation are shown in Fig. 3, and the jump conditions are

$$
\begin{gathered}
\tau_{Y Z}^{m^{+}}\left(X, H^{+}\right)-\tau_{Y Z}^{m^{-}}\left(X, H^{-}\right)=-f_{z} \delta\left(X-H^{\prime}\right), \\
w^{m^{+}}\left(X, H^{+}\right)-w^{m^{-}}\left(X, H^{-}\right)=-b_{z} U\left(X-H^{\prime}\right),
\end{gathered}
$$

where

$$
\begin{aligned}
& H^{\prime}=\alpha_{m} h+\sum_{k=1}^{m-1}\left(\alpha_{k}-\alpha_{k+1}\right) h_{k}, \\
& H=\beta_{m} h+\sum_{k=1}^{m-1}\left(\beta_{k}-\beta_{k+1}\right) h_{k} .
\end{aligned}
$$

Here $\tau_{Y Z}^{m^{+}}$and $\tau_{Y Z}^{m^{-}}$indicate the shear stress above and below the applied loadings, respectively, in the $m$ th layer (see Fig. 3). The location of the applied loadings is shifted by an amount of $H^{\prime}$ in the horizontal direction. The stress displacement relations expressed in the $(X, Y)$ coordinates within each layer become

$$
\begin{gathered}
\tau_{X Z}^{j}(X, Y)=\mu_{j}^{e} \frac{\partial w^{j}(X, Y)}{\partial X}, \\
\tau_{Y Z}^{j}(X, Y)=\mu_{j}^{e} \frac{\partial w^{j}(X, Y)}{\partial Y} .
\end{gathered}
$$

The boundary value problem described by Eqs. (16)-(20) is similar to the multilayered problem for an isotropic material. Hence the linear coordinate transformation presented in Eq. (15) changes the original anisotropic multilayered problem to the corresponding isotropic multilayered problem with a similar geometric configuration and boundary conditions. In the next section, the boundary value problem described by Eqs. (16)-(20) will be solved, and the relationships between the shear stresses in the $(x, y)$ and $(X, Y)$ coordinates established in Eq. (10) will be used to obtain the solutions for the original anisotropic multilayered problem.

\section{Fundamental Solutions in the Transformed Domain}

The boundary value problem of the previous section can be solved by the integral transform technique. The expressions for the field variables will be found by applying a Fourier transform over the spatial coordinate $X$ with parameter $\omega$. Take the Fourier transform pairs defined as

$$
\begin{gathered}
\widetilde{g}(\omega, Y)=\int_{-\infty}^{\infty} g(X, Y) e^{-i \omega X} d X, \\
g(X, Y)=\frac{1}{2 \pi} \int_{-\infty}^{\infty} \widetilde{g}(\omega, Y) e^{i \omega X} d \omega,
\end{gathered}
$$

and apply to the governing Eq. (16). Then, Eq. (16) becomes an ordinary differential equation with the following general solution in the Fourier transformed domain:

$$
\left[\begin{array}{c}
\widetilde{w}^{j} \\
\widetilde{\tau}_{Y Z}^{j}
\end{array}\right]=\left[\begin{array}{cc}
e^{\omega Y} & e^{-\omega Y} \\
\mu_{j}^{e} \omega e^{\omega Y} & -\mu_{j}^{e} \omega e^{-\omega Y}
\end{array}\right]\left[\begin{array}{l}
c_{j} \\
d_{j}
\end{array}\right] .
$$

Here $c_{j}$ and $d_{j}$ are undetermined coefficients for each layer and can be obtained by applying the boundary, continuity and jump conditions. Substituting the general solution into the continuity conditions at the interfaces, the recurrence relation for the coefficients for each layer can be expressed as

$$
\left[\begin{array}{c}
c_{j+1} \\
d_{j+1}
\end{array}\right]=\frac{1}{s_{j}^{\prime}} \mathbf{G}^{+}(j)\left[\begin{array}{c}
c_{j} \\
d_{j}
\end{array}\right],
$$

or

$$
\left[\begin{array}{c}
c_{j} \\
d_{j}
\end{array}\right]=\frac{1}{s_{j}^{\prime \prime}} \mathbf{G}^{-}(j)\left[\begin{array}{c}
c_{j+1} \\
d_{j+1}
\end{array}\right]
$$

where

$$
\begin{gathered}
\mathbf{G}^{+}(j)=\left[\begin{array}{cc}
1 & t_{j} e^{-2 \omega H_{j}} \\
t_{j} e^{2 \omega H_{j}} & 1
\end{array}\right], \\
\mathbf{G}^{-}(j)=\left[\begin{array}{cc}
1 & -t_{j} e^{-2 \omega H_{j}} \\
-t_{j} e^{2 \omega H_{j}} & 1
\end{array}\right], \\
t_{j}=\frac{\mu_{j+1}^{e}-\mu_{j}^{e}}{\mu_{j}^{e}+\mu_{j+1}^{e}}, \quad s_{j}^{\prime}=\frac{2 \mu_{j+1}^{e}}{\mu_{j}^{e}+\mu_{j+1}^{e}}, \quad s_{j}^{\prime \prime}=\frac{2 \mu_{j}^{e}}{\mu_{j}^{e}+\mu_{j+1}^{e}} .
\end{gathered}
$$

Here $t_{j}$ is called the reflection coefficient and $s_{j}^{\prime}, s_{j}^{\prime \prime}$ are the refraction coefficients. By applying the boundary condition for the top layer $\widetilde{\tau}_{Y Z}^{1}(\omega, 0)=0, c_{1}=d_{1}$ is obtained, and along with Eq. (22), the coefficients for the $m$ th layer can be related to those of the first layer as follows:

$$
\left[\begin{array}{l}
c_{m}^{+} \\
d_{m}^{+}
\end{array}\right]=\prod_{k=1}^{m-1} \frac{1}{s_{k}^{\prime}} \mathbf{G}^{+}(m-k)\left[\begin{array}{l}
c_{1} \\
c_{1}
\end{array}\right]
$$

where

$$
\prod_{k=1}^{n} a_{k}=a_{1} \cdot a_{2} \cdots a_{n} .
$$

Similarly, by applying the boundary condition for the bottom layer $\widetilde{\tau}_{Y Z}^{n}\left(\omega, H_{n}\right)=0, c_{n}=d_{n} e^{-2 \omega H_{n}}$ is obtained, and along with Eq. (23), the coefficients for the $m$ th layer can be represented by

$$
\left[\begin{array}{c}
c_{m}^{-} \\
d_{m}^{-}
\end{array}\right]=\prod_{k=m}^{n-1} \frac{1}{s_{k}^{\prime \prime}} \mathbf{G}^{-}(k)\left[\begin{array}{c}
d_{n} e^{-2 \omega H_{n}} \\
d_{n}
\end{array}\right] .
$$

Finally, the jump conditions in the $m$ th layer in the transformed domain are employed

$$
\begin{gathered}
c_{m}^{+} e^{\omega H}+d_{m}^{+} e^{-\omega H}-c_{m}^{-} e^{\omega H}-d_{m}^{-} e^{-\omega H}=\frac{i b_{z}}{\omega} e^{-i \omega H^{\prime}}, \\
c_{m}^{+} e^{\omega H}-d_{m}^{+} e^{-\omega H}-c_{m}^{-} e^{\omega H}+d_{m}^{-} e^{-\omega H}=\frac{-f_{z}}{\mu_{m}^{e} \omega} e^{-i \omega H^{\prime}},
\end{gathered}
$$

where $i=\sqrt{-1}$ and Eq. (26) can be written in a matrix form as

$$
\left[\begin{array}{cc}
e^{\omega H} & e^{-\omega H} \\
e^{\omega H} & -e^{-\omega H}
\end{array}\right]\left\{\left[\begin{array}{c}
c_{m}^{+} \\
d_{m}^{+}
\end{array}\right]-\left[\begin{array}{c}
c_{m}^{-} \\
d_{m}^{-}
\end{array}\right]\right\}=\left[\begin{array}{c}
\frac{i b_{z}}{\omega} \\
\frac{-f_{z}}{\mu_{m}^{e} \omega}
\end{array}\right] e^{-i \omega H^{\prime}} .
$$

For convenience, set $c_{m}^{+}=A_{1} c_{1}, d_{m}^{+}=A_{2} c_{1}, c_{m}^{-}=B_{1} d_{n}$ and $d_{m}^{-}$ $=B_{2} d_{n}$, where $A_{1}, A_{2}, B_{1}$ and $B_{2}$ are constants which can be expressed in matrix form as

$$
\left[\begin{array}{l}
A_{1} \\
A_{2}
\end{array}\right]=\prod_{k=1}^{m-1} \frac{1}{s_{k}^{\prime}} \mathbf{G}^{+}(m-k)\left[\begin{array}{l}
1 \\
1
\end{array}\right], \quad\left[\begin{array}{l}
B_{1} \\
B_{2}
\end{array}\right]=\prod_{k=m}^{n-1} \frac{1}{s_{k}^{\prime \prime}} \mathbf{G}^{-}(k)\left[\begin{array}{c}
e^{-2 \omega H_{n}} \\
1
\end{array}\right] .
$$


Therefore, Eq. (27) can be reformulated and coefficients $c_{1}$ and $d_{n}$ are found as follows:

$$
\begin{aligned}
{\left[\begin{array}{l}
c_{1} \\
d_{n}
\end{array}\right]=} & \frac{1 / 2}{A_{1} B_{2}-A_{2} B_{1}}\left[\begin{array}{ll}
B_{2} & -B_{1} \\
A_{2} & -A_{1}
\end{array}\right]\left[\begin{array}{cc}
e^{-\omega H} & e^{-\omega H} \\
e^{\omega H} & -e^{\omega H}
\end{array}\right] \\
& \times\left[\begin{array}{c}
\frac{i b_{z}}{\omega} \\
\frac{-f_{z}}{\mu_{m}^{e} \omega}
\end{array}\right] e^{-i \omega H^{\prime}} .
\end{aligned}
$$

The undetermined constants $c_{j}$ and $d_{j}$ for each layer are obtained with the aid of the recurrence relations given in Eqs. (22) and (23). After substituting the coefficients $c_{j}$ and $d_{j}$ into Eq. (21), the full-field solutions for each layer are completely determined in the transformed domain. Omitting the lengthy algebraic derivation, the general solutions for each layer are finally expressed as

$$
\begin{aligned}
{\left[\begin{array}{c}
\widetilde{w}^{j} \\
\widetilde{\tau}_{Y Z}^{j}
\end{array}\right]=} & \frac{-e^{-i \omega H^{\prime}}}{2\left(A_{2} B_{1}-A_{1} B_{2}\right) \omega \mu_{m}^{e}}\left(B_{2} e^{-\omega H}\left(f_{z}-i \mu_{m}^{e} b_{z}\right)\right. \\
& \left.+B_{1} e^{\omega H}\left(f_{z}+i \mu_{m}^{e} b_{z}\right)\right) \cdot\left[\begin{array}{cc}
e^{\omega Y} & e^{-\omega Y} \\
\mu_{j}^{e} \omega e^{\omega Y} & -\mu_{j}^{e} \omega e^{-\omega Y}
\end{array}\right] \\
& \times\left\{\prod_{k=1}^{j-1} \frac{1}{s_{k}^{\prime}}\left[\begin{array}{cc}
1 & t_{j-k} e^{-2 \omega H_{j-k}} \\
t_{j-k} e^{2 \omega H_{j-k}} & 1
\end{array}\right]\right\}\left[\begin{array}{l}
1 \\
1
\end{array}\right],
\end{aligned}
$$

for $1 \leqslant j \leqslant m$. And for $m \leqslant j \leqslant n$,

$$
\begin{aligned}
{\left[\begin{array}{c}
\widetilde{w}^{j} \\
\widetilde{\tau}_{Y Z}^{j}
\end{array}\right]=} & \frac{-e^{-i \omega H^{\prime}}}{2\left(A_{2} B_{1}-A_{1} B_{2}\right) \omega \mu_{m}^{e}}\left(A_{2} e^{-\omega H}\left(f_{z}-i \mu_{m}^{e} b_{z}\right)\right. \\
& \left.+A_{1} e^{\omega H}\left(f_{z}+i \mu_{m}^{e} b_{z}\right)\right) \cdot\left[\begin{array}{cc}
e^{\omega Y} & e^{-\omega Y} \\
\mu_{j}^{e} \omega e^{\omega Y} & -\mu_{j}^{e} \omega e^{-\omega Y}
\end{array}\right] \\
& \times\left\{\prod_{k=j}^{n-1} \frac{1}{s_{k}^{\prime \prime}}\left[\begin{array}{cc}
1 & -t_{k} e^{-2 \omega H_{k}} \\
-t_{k} e^{2 \omega H_{k}} & 1
\end{array}\right]\right\}\left[\begin{array}{c}
e^{-2 \omega H_{n}} \\
1
\end{array}\right] .
\end{aligned}
$$

\section{Full-Field Solutions and Their Physical Meaning}

The complete solutions in the Fourier transformed domain for multilayered media have been presented in Eqs. (30) and (31) in the previous section. The solutions are so complicated that it is not easy to obtain the inverse Fourier transform. In order to construct the explicit analytical full-field solution of the $n$-layered medium, the Taylor series expansion is used in this study.

Because of the denominators in Eqs. (29)-(31), it is impossible to invert the Fourier transform directly. By examining the structure of the denominator of Eq. (29), both the numerator and denominator are multiplied by a constant $S=\left(\prod_{k=1}^{m-1} s_{k}^{\prime}\right)\left(\prod_{k=m}^{n-1} s_{k}^{\prime \prime}\right)$.

Then it becomes

$$
\begin{aligned}
{\left[\begin{array}{l}
c_{1} \\
d_{n}
\end{array}\right]=} & \frac{S / 2}{S\left(A_{1} B_{2}-A_{2} B_{1}\right) \mu_{m}^{e} \omega}\left[\begin{array}{cc}
B_{2} & -B_{1} \\
A_{2} & -A_{1}
\end{array}\right]\left[\begin{array}{cc}
e^{-\omega H} & e^{-\omega H} \\
e^{\omega H} & -e^{\omega H}
\end{array}\right] \\
& \times\left[\begin{array}{c}
i \mu_{m}^{e} b_{z} \\
-f_{z}
\end{array}\right] e^{-i \omega H^{\prime}} .
\end{aligned}
$$

The denominator in Eq. (32), $S\left(A_{1} B_{2}-A_{2} B_{1}\right)$, can be decomposed into the form of $(1-p)$ where $p=1-S\left(A_{1} B_{2}-A_{2} B_{1}\right)$. It can be shown that $p<1$ for $\omega>0$. By a series expansion, we obtain $1 / 1-p=\sum_{l=0}^{\infty} p^{l}$ so that Eq. (32) can be rewritten as

$$
\left[\begin{array}{l}
c_{1} \\
d_{n}
\end{array}\right]=\frac{S}{2 \omega}\left[\begin{array}{ll}
B_{2} & -B_{1} \\
A_{2} & -A_{1}
\end{array}\right]\left[\begin{array}{cc}
e^{-\omega H} & e^{-\omega H} \\
e^{\omega H} & -e^{\omega H}
\end{array}\right]\left[\begin{array}{c}
i b_{z} \\
\frac{-f_{z}}{\mu_{m}^{e}}
\end{array}\right] e^{-i \omega H^{\prime}} \cdot \sum_{l=0}^{\infty} p^{l}
$$

Now, the solutions for $c_{1}$ and $d_{n}$ are linear combinations of exponential functions, i.e., $M_{i} e^{ \pm f\left(\omega, H_{i}\right)}$, and so are $c_{j}$ and $d_{j}$. Note that $M_{i}$ are constants which denote the magnitude of the exponential function, and $f\left(\omega, H_{i}\right)$ are functions of $\omega$ and $H_{i}$.

The solutions for the displacement and shear stress in Eqs. (30) and (31) can also be expressed in similar forms. By a complicated algebraic derivation, the explicit expressions for displacement and shear stress in the Fourier transformed domain are obtained as follows:

$$
\begin{gathered}
\widetilde{w}^{j}=\sum_{l=0}^{\infty} \sum_{k=1}^{N} \frac{M_{k}}{\mu_{m}^{e} \omega}\left[\begin{array}{l}
-\left(f_{z}-i \mu_{m}^{e} b_{z}\right) e^{\omega\left(Y-H+F_{k}^{c^{\prime}}\right)}-\left(f_{z}+i \mu_{m}^{e} b_{z}\right) e^{\omega\left(Y+H+F_{k}^{c^{\prime \prime}}\right)} \\
-\left(f_{z}-i \mu_{m}^{e} b_{z}\right) e^{-\omega\left(Y+H-F_{k}^{d^{\prime}}\right)}-\left(f_{z}+i \mu_{m}^{e} b_{z}\right) e^{-\omega\left(Y-H-F_{k}^{d^{\prime \prime}}\right)}
\end{array}\right] e^{-i \omega H^{\prime}}, \\
\widetilde{\tau}_{Y Z}^{j}=\sum_{l=0}^{\infty} \sum_{k=1}^{N} \frac{\mu_{j}^{e} M_{k}}{\mu_{m}^{e}}\left[\begin{array}{l}
-\left(f_{z}-i \mu_{m}^{e} b_{z}\right) e^{\omega\left(Y-H+F_{k}^{c^{\prime}}\right)}-\left(f_{z}+i \mu_{m}^{e} b_{z}\right) e^{\omega\left(Y+H+F_{k}^{c^{\prime \prime}}\right)} \\
+\left(f_{z}-i \mu_{m}^{e} b_{z}\right) e^{-\omega\left(Y+H-F_{k}^{d^{\prime}}\right)}+\left(f_{z}+i \mu_{m}^{e} b_{z}\right) e^{-\omega\left(Y-H-F_{k}^{d^{\prime \prime}}\right)}
\end{array}\right] e^{-i \omega H^{\prime}},
\end{gathered}
$$

where

$$
\begin{cases}N=2^{n+j-m-1} \cdot\left(2^{n}-1\right)^{l}, & 1 \leqslant j \leqslant m, \\ N=2^{n+m-j-1} \cdot\left(2^{n}-1\right)^{l}, & m \leqslant j \leqslant n .\end{cases}
$$

Here $n$ is the number of layers, $m$ denotes the layer that is subjected to the applied loading, and $j$ is the $j$ th layer where the solution is required. The terms $M_{k}, F_{k}^{c^{\prime}}, F_{k}^{c^{\prime \prime}}, F_{k}^{d^{\prime}}$, and $F_{k}^{d^{\prime \prime}}$ in Eq. (34) are very complicated and difficult to present. The following functions are first defined as

$$
\begin{gathered}
\begin{cases}a_{1}=1, f_{1}^{A}=0, & k=1,2 \cdots m-1, \\
a_{i+2^{k-1}}=a_{i} t_{k}, & i=1,2 \cdots 2^{k-1}, \\
f_{i+2^{k-1}}^{A}=-\left(f_{i}^{A}+2 H_{k}\right),\end{cases} \\
\begin{cases}b_{1}=1, f_{1}^{B_{1}}=-2 H_{n}, f_{1}^{B_{2}}=0, \\
b_{i+2^{k-1}}=-b_{i} t_{n-k}, & k=1,2 \cdots n-m, \\
f_{i+2^{k-1}}^{B_{1}}=-\left(f_{i}^{B_{1}}+2 H_{n}+2 H_{n-k}\right), & i=1,2 \cdots 2^{k-1}, \\
f_{i+2^{k-1}}^{B_{2}}=-\left(f_{i}^{B_{2}}+2 H_{n}-2 H_{n-k}\right),\end{cases}
\end{gathered}
$$




$$
\begin{gathered}
\left\{\begin{array}{lll}
r_{(i-1) \cdot 2^{n-m}+k}^{p}=-a_{i} b_{k}, & r_{2^{n-1}+(i-1) \cdot 2^{m-1}+k}^{p}=a_{i} b_{k}, & i=1,2, \cdots 2^{m-1}, \\
g_{(i-1) \cdot 2^{n-m}+k}^{p}=f_{i}^{A}+f_{k}^{B_{2}}, & g_{2^{n-1}+(k-1) \cdot 2^{m-1}+i}^{p}=f_{k}^{B_{1}}-f_{i}^{A}, & k=1,2, \cdots 2^{n-m},
\end{array}\right. \\
\left\{\begin{array}{cc}
r_{k}^{l}=\prod_{0=1}^{l} r_{l_{0}}^{p}, & i_{1}, i_{2}, 3 \\
g_{k}^{l}=\sum_{0=1}^{l} g_{l_{0}}^{p}, & k=\sum_{0=1}^{l-1}\left(i_{0}-2\right)\left(2^{n}-1\right)+\left(i_{l}-1\right),
\end{array}\right.
\end{gathered}
$$

for $l=0, r_{i_{0}}^{0}=1$ and $g_{i_{0}}^{0}=0$.

For the case of $1 \leqslant j \leqslant m$, the following functions are defined:

$$
\left\{\begin{array}{l}
f_{(i-1) \cdot 2^{n-m}+k}^{c^{\prime}}=f_{k}^{B_{2}}+f_{i}^{A}, \\
f_{(i-1) \cdot 2^{n-m}+k}^{c^{\prime \prime}}=f_{k}^{B_{1}}+f_{i}^{A}, \quad i=1,2, \cdots 2^{j-1}, \\
f_{(i-1) \cdot 2^{n-m}+k}^{d^{\prime}}=f_{k}^{B_{2}}-f_{i}^{A}, \quad k=1,2, \cdots 2^{n-m} . \\
f_{(i-1) \cdot 2^{n-m}+k}^{d^{\prime \prime}}=f_{k}^{B_{1}}-f_{i}^{A},
\end{array}\right.
$$

Finally, the terms $M_{k}, F_{k}^{c^{\prime}}, F_{k}^{c^{\prime \prime}}, F_{k}^{d^{\prime}}$, and $F_{k}^{d^{\prime \prime}}$ indicated in Eq. (34) can be expressed explicitly as

$$
\begin{cases}M_{(k-1) \cdot 2^{n+j-m-1}+i}=\frac{-1}{2}\left(\prod_{0=j}^{m-1} S_{0}^{\prime}\right) r_{k}^{l} r_{i}^{p}, & \\ F_{(k-1) \cdot 2^{n+j-m-1}+i}^{c^{\prime}}=g_{k}^{l}+f_{i}^{c^{\prime}}, & k=1,2, \cdots\left(2^{n}-1\right)^{l}, \\ F_{(k-1) \cdot 2^{n+j-m-1}+i}^{c^{\prime \prime}}=g_{k}^{l}+f_{i}^{c^{\prime \prime}}, & i=1,2, \cdots 2^{n+j-m-1} . \\ F_{(k-1) \cdot 2^{n+j-m-1}+i}^{d^{\prime}}=g_{k}^{l}+f_{i}^{d^{\prime}}, & \\ F_{(k-1) \cdot 2^{n+j-m-1}+i}^{d^{\prime \prime}}=g_{k}^{l}+f_{i}^{d^{\prime \prime}}, & \end{cases}
$$

For the case of $m \leqslant j \leqslant n$, the expressions are

$$
\left\{\begin{array}{l}
f_{(i-1) \cdot 2^{m-1}+k}^{c^{\prime}}=f_{i}^{B_{1}}+f_{k}^{A}, \\
f_{(i-1) \cdot 2^{m-1}+k}^{c^{\prime \prime}}=f_{i}^{B_{1}}+f_{k}^{A}, \quad i=1,2, \cdots 2^{n-j}, \\
f_{(i-1) \cdot 2^{m-1}+k}^{d^{\prime}}=f_{i}^{B_{2}}-f_{k}^{A}, \quad k=1,2, \cdots 2^{m-1} . \\
f_{(i-1) \cdot 2^{m-1}+k}^{d^{\prime \prime}}=f_{i}^{B_{2}}+f_{k}^{A},
\end{array}\right.
$$

The terms $M_{k}, F_{k}^{c^{\prime}}, F_{k}^{c^{\prime \prime}}, F_{k}^{d^{\prime}}$, and $F_{k}^{d^{\prime \prime}}$ are presented by

$$
\begin{cases}M_{(k-1) \cdot 2^{n-j+m-1}+i}=\frac{1}{2}\left(\prod_{0=m}^{j-1} S_{0}^{\prime \prime}\right) r_{k}^{l} r_{2^{n-1}+i}^{p}, & \\ F_{(k-1) \cdot 2^{n-j+m-1}+i}^{c^{\prime}}=g_{k}^{l}+f_{i}^{c^{\prime}}, & k=1,2, \cdots\left(2^{n}-1\right)^{l}, \\ F_{(k-1) \cdot 2^{n-j+m-1}+i}^{c^{\prime \prime}}=g_{k}^{l}+f_{i}^{c^{\prime \prime}}, & i=1,2, \cdots 2^{n+m-j-1} . \\ F_{(k-1) \cdot 2^{n-j+m-1}+i}^{d^{\prime}}=g_{k}^{l}+f_{i}^{d^{\prime}}, & \\ F_{(k-1) \cdot 2^{n-j+m-1}+i}^{d^{\prime \prime}}=g_{k}^{l}+f_{i}^{d^{\prime \prime}}, & \end{cases}
$$

The structures of the complete solutions given in Eq. (34) have some interesting characteristics. The solutions are composed of infinite terms, and it is interesting to note that each term represents the solution in the transformed domain for a concentrated loading in an infinite homogeneous medium. The term with $\left(Y \pm H \pm F_{k}\right)$ in the exponential functions indicates the location of the loading. However, $M_{k} b_{z}$ and $\mu_{j}^{e} / \mu_{m}^{e} M_{k} f_{z}$ represent the magnitudes of the concentrated dislocation and force, respectively. $F_{k}$ is dependent on the locations of the interfaces, i.e., $H_{j}$, and $M_{k}$ depends only on the reflection and refraction coefficients, i.e., $t_{j}, s_{j}^{\prime}$, and $s_{j}^{\prime \prime}$.

Only one term in the infinite series of Eq. (34) represents the applied concentrated force $f_{z}$ and dislocation $b_{z}$ in an infinite medium at $Y=H$ and $X=H^{\prime}$, all the remaining terms are image forces and dislocations that are induced to satisfy the boundary and interface conditions. This method is referred to as the method of images. The advantage of this method is that the solutions of problems with complicated geometric configurations can be constructed by superposing the solution in an infinite medium. The mathematical derivation in this section provides an automatic determination for the locations and magnitudes of all the image forces.

Since the solutions in the transformed domain expressed in Eq 
(34) are exponential functions of $\omega$, only two different inverse Fourier transformations for exponential functions are required, which are

$$
\begin{gathered}
\int_{-\infty}^{\infty} e^{-\omega\left(Y \pm H \pm F_{k}\right)} \cdot e^{-i \omega H^{\prime}} \cdot e^{i \omega X} d \omega \\
=\frac{1}{\pi} \frac{Y \pm H \pm F_{k}}{\left(X-H^{\prime}\right)^{2}+\left(Y \pm H \pm F_{k}\right)^{2}},
\end{gathered}
$$

$$
\begin{aligned}
\int_{-\infty}^{\infty} & \frac{1}{\omega} e^{-\omega\left(Y \pm H \pm F_{k}\right)} \cdot e^{-i \omega H^{\prime}} \cdot e^{i \omega X} d \omega \\
= & \frac{-1}{2 \pi} \ln \left[\left(X-H^{\prime}\right)^{2}+\left(Y \pm H \pm F_{k}\right)^{2}\right] .
\end{aligned}
$$

Therefore, the inverse Fourier transformation for Eq. (34) can be easily derived and the results are

$$
\begin{aligned}
& w^{j}=\sum_{l=0}^{\infty} \sum_{k=1}^{N} \frac{M_{k}}{\pi}\left\{\begin{array}{l}
b_{z}\left[\begin{array}{l}
-\tan ^{-1} \frac{Y-H+F_{k}^{c^{\prime}}}{X-H^{\prime}}+\tan ^{-1} \frac{Y+H+F_{k}^{c^{\prime \prime}}}{X-H^{\prime}} \\
+\tan ^{-1} \frac{Y+H+F_{k}^{d^{\prime}}}{X-H^{\prime}}-\tan ^{-1} \frac{Y-H-F_{k}^{d^{\prime \prime}}}{X-H^{\prime}}
\end{array}\right] \\
+\frac{f_{z}}{2 \mu_{m}^{e}}\left[\begin{array}{c}
\ln \left(\left(X-H^{\prime}\right)^{2}+\left(Y-H+F_{k}^{c^{\prime}}\right)^{2}\right)+\ln \left(\left(X-H^{\prime}\right)^{2}+\left(Y+H+F_{k}^{c^{\prime \prime}}\right)^{2}\right) \\
+\ln \left(\left(X-H^{\prime}\right)^{2}+\left(Y+H-F_{k}^{d^{\prime}}\right)^{2}\right)+\ln \left(\left(X-H^{\prime}\right)^{2}+\left(Y-H-F_{k}^{d^{\prime \prime}}\right)^{2}\right)
\end{array}\right]
\end{array}\right\}, \\
& \tau_{Y Z}^{j}=\sum_{l=0}^{\infty} \sum_{k=1}^{N} \frac{\mu_{j}^{e} M_{k}}{\pi} b_{b_{z}\left[\begin{array}{c}
\frac{-\left(X-H^{\prime}\right)}{\left(X-H^{\prime}\right)^{2}+\left(Y-H+F_{k}^{c^{\prime}}\right)^{2}}+\frac{\left(X-H^{\prime}\right)}{\left(X-H^{\prime}\right)^{2}+\left(Y+H+F_{k}^{c^{\prime \prime}}\right)^{2}} \\
+\frac{\left(X-H^{\prime}\right)}{\left(X-H^{\prime}\right)^{2}+\left(Y+H-F_{k}^{d^{\prime}}\right)^{2}}-\frac{\left(X-H^{\prime}\right)}{\left(X-H^{\prime}\right)^{2}+\left(Y-H-F_{k}^{d^{\prime \prime}}\right)^{2}}
\end{array}\right]}^{+\frac{Y-H+F_{k}^{c^{\prime}}}{\mu_{m}^{e}}\left[\begin{array}{l}
\frac{Y+H+F_{k}^{c^{\prime}}}{\left(X-H^{\prime}\right)^{2}+\left(Y-H+F_{k}^{c^{\prime}}\right)^{2}}+\frac{\left.Y-H^{\prime}\right)^{2}+\left(Y+H+F_{k}^{c^{\prime \prime}}\right)^{2}}{\left(X-F_{k}^{d^{\prime \prime}}\right.} \\
\frac{Y+H-F_{k}^{d^{\prime}}}{\left(X-H^{\prime}\right)^{2}+\left(Y+H-F_{k}^{d^{\prime}}\right)^{2}}+\frac{Y-H-F^{d^{\prime}}}{\left(X-H^{\prime}\right)^{2}+\left(Y-H-F_{k}^{d^{\prime \prime}}\right)^{2}}
\end{array}\right] .}
\end{aligned}
$$

Finally, by substituting $X$ and $Y$ defined in Eq. (15) into Eq. (37), and using the displacement and stress relations in Eq. (10), the complete solutions for the original problem of the anisotropic multilayered medium can be obtained. Equation (37) is the explicit expression of the Green's function for the multilayered medium subjected to the antiplane shear deformation.

\section{Numerical Examples and Discussions}

The full-field analysis of shear stresses in a layered medium consisting of 12 layers subjected to interior forces is presented in this section. In the analysis of a nonhomogeneous multilayered medium, it is sometimes the practice to treat the multilayered medium as a single homogeneous layer with effective or homogenized stiffness constants. The differences in the stress distributions between layered medium with 12 layers and one homogeneous layer with the effective material constants obtained from the averaged material properties of 12 layers will be discussed in detail. The effective stiffness constants of one homogeneous layer, $\widetilde{C}_{i j}$, are obtained from the homogenized weighted properties of the 12 layers using the formula

$$
\widetilde{C}_{i j}=\frac{\sum_{k=2}^{n} C_{i j}^{k}\left(h_{k}-h_{k-1}\right)+C_{i j}^{1} h_{1}}{h_{n}} .
$$

As indicated in Eq. (38), the effective stiffness constants are obtained by averaging the stiffness constants over the thickness of each layer. The complete explicit expressions for the displacement and shear stress given in Eqs. (35) and (37) are used to construct the program for numerical calculations. For the 12-layered me- dium, the thickness of each layer is the same and equal to 1 , and the elastic stiffness constants for each layer are listed as follows:

$$
\begin{aligned}
{\left[C_{44}^{1}, C_{45}^{1}, C_{55}^{1} ; C_{44}^{2}, C_{45}^{2}, C_{55}^{2} ; \ldots ; C_{44}^{12}, C_{45}^{12}, C_{55}^{12}\right] } \\
=[3960,0,7170 ; 7170,1220,3960 ; 3960, \\
\quad-1220,7170 ; 3585,610,3960 ; 3960, \\
\quad-610,3585 ; 4430,0,0,4430 ; 1980 ; 0,1980 ; 7170,1220,3585 ; \\
\\
\quad 5158,-610,3585 ; 3585,0,5185 ; 3960,0,3960 ; 6160, \\
\quad-2440,3330] \mathrm{GPa} .
\end{aligned}
$$

The effective elastic constants for one homogeneous layer obtained from Eq. (38) are

$$
\left[\widetilde{C}_{44}, \widetilde{C}_{45}, \widetilde{C}_{55}\right]=[4347,-169,4131] \mathrm{GPa} .
$$

An antiplane body force $f_{z}$ is applied within the seventh layer at $x=0$ and $y=6.5$. The distributions of the shear stress $\tau_{y z}$ along the $y$-axis at $x=0$ and $x=2$ are shown in Figs. 4 and 5, respectively. In addition, the $\tau_{x z}$ stress distributions along the $y$-axis at $x=0$ and $x=2$ are displayed in Figs. 6 and 7, respectively. To generate the shear stress distributions, the series solution is truncated if the numerical calculation is within the accuracy of 0.1 percent.

As shown in Figs. 4 and 5, the multilayered solution for $\tau_{y z}$ is continuous at the interfaces and approaches zero at the top and bottom boundaries. The results of $\tau_{y z}$ for multilayered solutions and one homogeneous layer solution are closely related at $x=0$, and only a small difference appears at $x=2$. This implies that the 


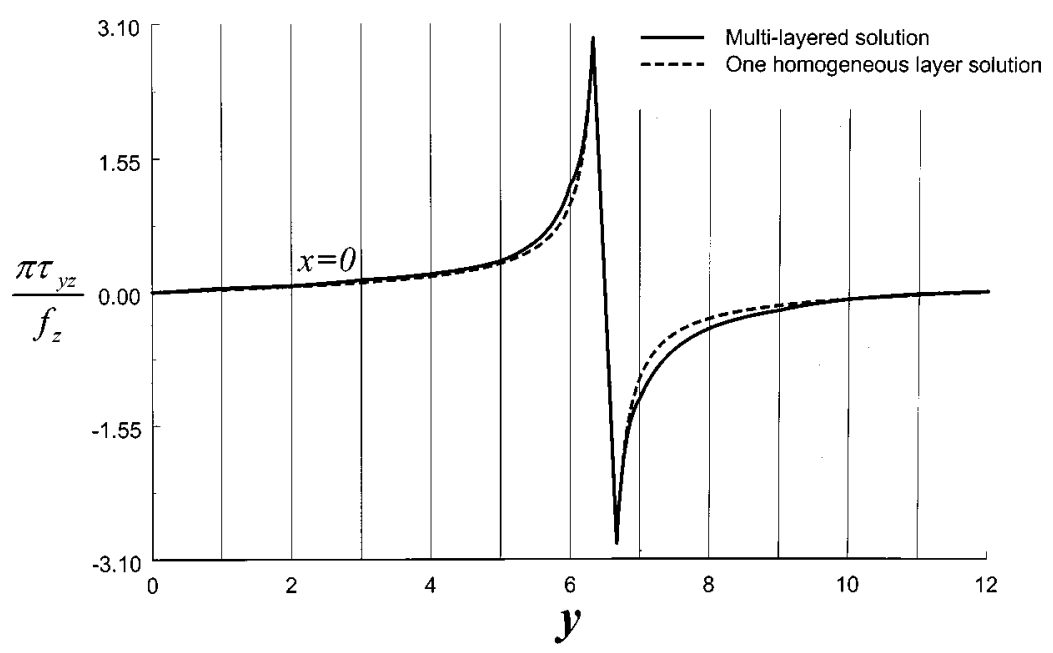

Fig. 4 Distribution of $\tau_{y z}$ at $x=0$ along $y$-axis for the 12-layered medium and one homogeneous layer

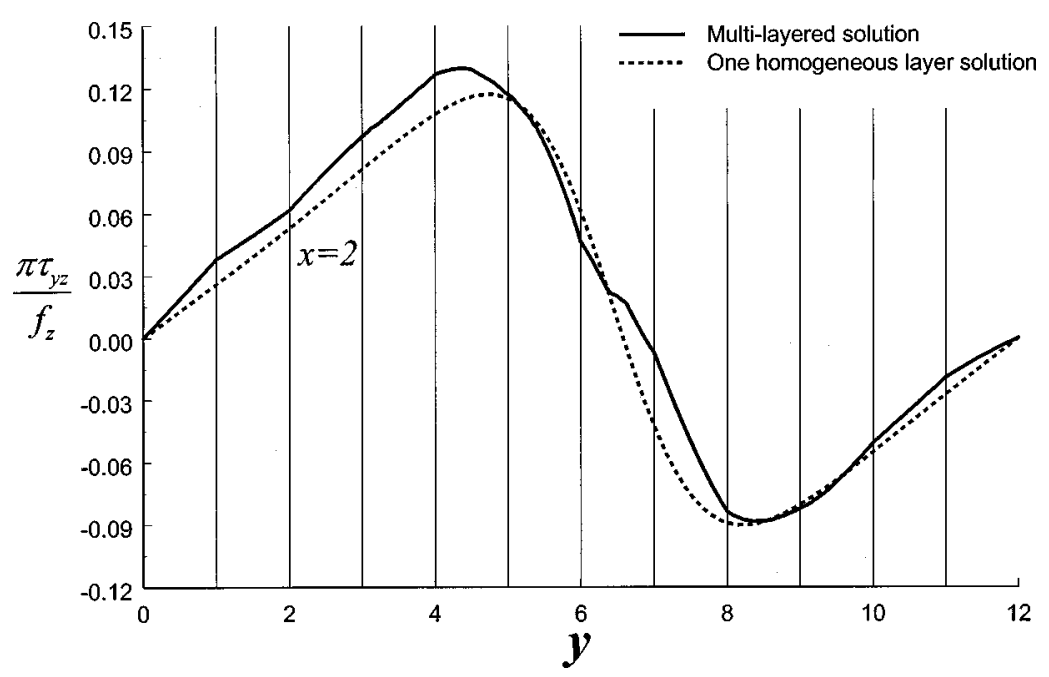

Fig. 5 Distribution of $\tau_{y z}$ at $x=2$ along $y$-axis for the 12-layered medium and one homogeneous layer

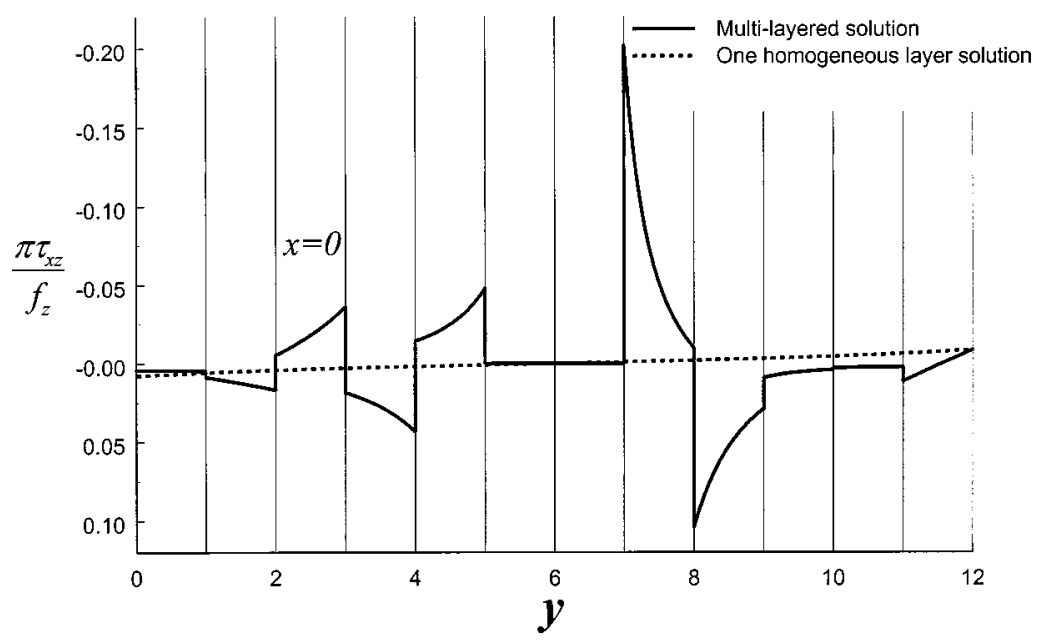

Fig. 6 Distribution of $\tau_{x z}$ at $x=0$ along $y$-axis for the 12-layered medium and one homogeneous layer 


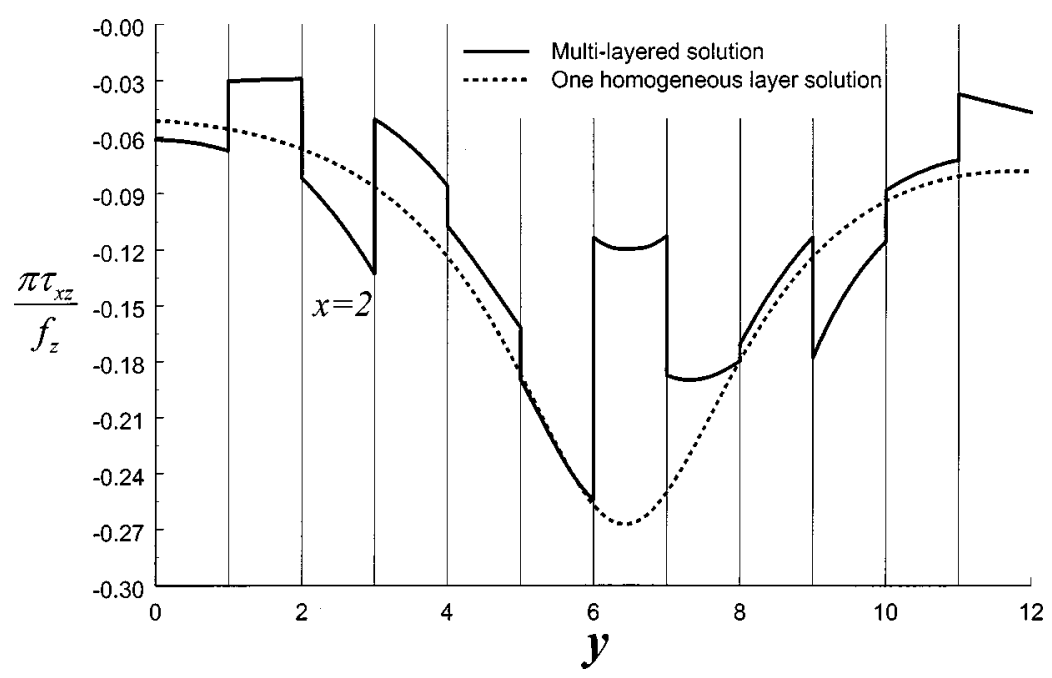

Fig. 7 Distribution of $\tau_{x z}$ at $x=2$ along $y$-axis for the 12-layered medium and one homogeneous layer

shear stress $\tau_{y z}$ of the multilayered problem can be obtained with good approximation from the result of a much simpler one homogeneous layer solution. As indicated in Figs. 6 and 7, since $\tau_{x z}$ is discontinuous at the interfaces for the multilayered solution, the results for multilayered solution and one homogeneous layer solution differ substantially. This indicates that it is not suitable to use the single homogeneous layer solution to simulate the multilayered problem for the shear stress $\tau_{x z}$.

\section{Concluding Remarks}

An analytical investigation of multilayered anisotropic media subjected to concentrated forces and screw dislocations has been provided in this study. A general linear coordinate transformation for multilayered media was introduced to simplify the governing equilibrium equation without complicating the boundary and interface continuity conditions. With this linear coordinate transformation, the original anisotropic multilayered problem can be reduced to an equivalent isotropic multilayered problem. The linear coordinate transformation introduced in this study substantially reduces the dependence on elastic constants in the antiplane anisotropic multilayered media. By using the Fourier transform technique and a series expansion, analytical solutions for displacement and stresses are presented in an explicit form. The complete solutions for this complicated problem consist only of very simple solutions obtained from an infinite homogeneous medium with concentrated loadings. Except for the original applied loading, the remaining terms in the infinite series are image forces which are induced to satisfy the boundary and interface conditions. The mathematical approach introduced in this study provides an automatic determination for the locations and magnitudes of all the image forces. A computational program for numerical calculation of the full-field analysis is easily constructed by using the explicit formulation of the solutions. In order to simplify the problem, the nonhomogeneous multilayered medium is sometimes treated as a single homogeneous layer with effective material constants. Hence the numerical results for the multilayered problem were compared with the solutions obtained by considering the multilayered medium as a single homogeneous layer with effective material constants.

\section{Acknowledgments}

The financial support of the authors from the National Science Council, Republic of China, through Grant NSC 87-2218-E002-
022 to National Taiwan University is gratefully acknowledged. The authors also wish to express their great appreciation to the Associate Editor and reviewers for their valuable suggestions.

\section{References}

[1] Horgan, C. O., and Miller, K. L., 1994, “Antiplane Shear Deformations for Homogeneous and Inhomogeneous Anisotropic Linearly Elastic Solids," ASME J. Appl. Mech., 61, pp. 23-29.

[2] Ma, C. C., 1992, "Antiplane Problems of Monoclinic Material," ASCE J. Eng. Mech., 118, pp. 1765-1782.

[3] Ma, C. C., and Hour, B. L., 1989, "Analysis of Dissimilar Anisotropic Wedges Subjected to Antiplane Shear Deformation,'” Int. J. Solids Struct., 25, pp. 1295-1309.

[4] Retting, G., 1986, “'Anti-plane Stress Analysis of a Cracked Rectangular Orthotropic Beam,'” Eng. Fract. Mech., 23, pp. 441-454.

[5] Wu, K. C., and Chiu, Y. T., 1991, "Antiplane Shear Interface Cracks in Anisotropic Bimaterials,'” ASME J. Appl. Mech., 58, pp. 399-403.

[6] Ting, T. C. T., 1996, Anisotropic Elasticity, Oxford University Press, New York.

[7] Jiang, Q., and Knowles, J. K., 1991, "A Class of Compressible Elastic Materials Capable of Sustaining Finite Anti-plane Shear,' J. Elast., 25, pp. 193 201.

[8] Polignone, D. A., and Horgan, C. O., 1992, “Axisymmetric Finite Anti-plane Shear of Compressible Nonlinearly Elastic Circular Tubes,' Q. Appl. Math., 50, pp. 323-341.

[9] Horgan, C. O., 1995, "Anti-Plane Shear Deformations in Linear and Nonlinear Solid Mechanics,' SIAM Rev., 37, pp. 53-81.

[10] Lekhnitskii, S. G., 1963, Theory of Elasticity of an Anisotropic Body, HoldenDay, San Francisco, CA.

[11] Stroh, A. N., 1958, "Dislocations and Cracks in Anisotropic Elasticity,', Philos. Mag., 7, pp. 625-646.

[12] Ma, C. C., 1996, "Relationship of Anisotropic and Isotropic Materials for Antiplane Problems,"’ AIAA J., 34, pp. 2453-2456.

[13] Yang, W., and Ma, C. C., 1998, "Orthotropic Transform for Planar Anisotropic Elasticity and Reduced Dependence of Elastic Constants,' Proc. R. Soc. London, Ser. A, A454, pp. 1843-1855.

[14] Bufler, H., 1971, “Theory of Elasticity of a Multilayered Medium,'” J. Elast., 1, pp. 125-143.

[15] Bahar, L. Y., 1972, “'Transfer Matrix Approach to Layered Systems,'” J. Eng. Mech. Div., 98, pp. 1159-1172.

[16] Pindera, M.-J., 1991, “Local/Global Stiffness Matrix Formulation for Composite Materials and Structures," Composites Eng., 1, pp. 69-83.

[17] Pindera, M.-J., and Lane, M. S., 1993, "Frictionless Contact of Layered HalfPlanes, Part I: Analysis,' ASME J. Appl. Mech., 60, pp. 633-639.

[18] Pindera, M.-J., and Lane, M. S., 1993, "Frictionless Contact of Layered HalfPlanes, Part II: Numerical Results,”' ASME J. Appl. Mech., 60, pp. 640-645.

[19] Baxter, S. C., and Horgan, C. O., 1995, "End Effects for Anti-Plane Shear Deformations of Sandwich Structures," J. Elast., 40, pp. 123-164.

[20] Baxter, S. C., and Horgan, C. O., 1997, “Anti-Plane Shear Deformations of Anisotropic Sandwich Structures: End Effect,' Int. J. Solids Struct., 34, pp. 79-98.

[21] Wu, K. C., and Chiu, Y. T., 1996, "Antiplane Shear Analysis of a Semiinfinite Multi-layered Monoclinic Strip,” Acta Mech., 117, pp. 205-214. 